\title{
Fortaleciendo las competencias ciudadanas a través de ambientes lúdicos
}

\author{
Katherine Dayana Romero-Vaca ${ }^{1}$ \\ Universidad Pedagógica y Tecnológica de Colombia \\ katherine.romero@uptc.edu.co \\ Nancy Marcela Soriano-Vela ${ }^{2}$ \\ Universidad Pedagógica y Tecnológica de Colombia \\ nancy.soriano@uptc.edu.co \\ José Eriberto Cifuentes-Medina ${ }^{3}$ \\ Universidad Pedagógica y Tecnológica de Colombia \\ joseeriberto.cifuentes@uptc.edu.co
}

DOI: https://doi.org/10.21158/2357514x.v9.n1.2021.3056

Cómo citar este artículo: Romero-Vaca, K. D.; Soriano-Vela, N. M.: Cifuentes-Medina, J. E. (2021). Fortaleciendo las competencias ciudadanas a través de ambientes lúdicos. Revista Virtu@lmente, 9(1), Páginas. DOI: https://doi.org/10.21158/2357514x.v9.n1.2021.3056

Fecha de recepción: 02 de octubre de 2020

Fecha de aprobación: 12 de agosto de 2021

\section{Resumen}

El presente trabajo nace como una alternativa al observar una debilitada convivencia escolar entre estudiantes de segundo grado de una institución educativa. Durante la etapa de diagnóstico se determinó que esto se debía a la falta de uso de destrezas propias para la convivencia pacífica, por lo que se establece que es necesario fortalecer las competencias ciudadanas de los estudiantes, entendiendo que estas son un conjunto de habilidades que, relacionadas con conocimientos, permiten la formación de individuos capaces de reconocer su entorno y la forma de relacionarse con él. Para alcanzar este objetivo se acudió al uso de ambientes lúdicos, definidos como espacios que permiten la interacción entre las personas y motivan la expresión, la diversión y la reflexión. La estrategia se ejecutó por medio de la planeación y la aplicación de talleres pedagógicos que son analizados a través de diarios de campo con el fin de reconocer los avances obtenidos en estos. Organizar ambientes lúdicos mediante distintos talleres pedagógicos resultó enriquecedor, pues durante el desarrollo de

1 Licenciada en Educación Básica con énfasis en Matemáticas, Humanidades y Lengua Castellana - Universidad Pedagógica y Tecnológica de Colombia. ORCID: https://orcid.org/0000-0001-7209-0824

2 Licenciada en Educación Básica con énfasis en Matemáticas, Humanidades y Lengua Castellana - Universidad Pedagógica y Tecnológica de Colombia. ORCID: https://orcid.org/0000-0001-5237-7502

${ }^{3}$ Licenciado en Filosofía y Educación Religiosa y en Teología - Universidad Santo Tomas. Especialista en Evaluación Educativa Universidad Santo Tomas. Magíster en Educación - Universidad Santo Tomas. Estudiante de Doctorado - Universidad Santo Tomas. Licenciado en Ciencias Sociales y Especialista en Pedagogía y Docencia - Fundación Universitaria del Área Andina. Doctor en Educación - Universidad de Baja California. ORCID: http://orcid.org/0000-0001-5702-620X 
estas actividades se observaron situaciones de desacuerdo y discusiones que se solucionaron a través del diálogo, la reflexión y el trabajo en equipo, destrezas necesarias en las competencias ciudadanas. Finalmente, se constató que esta fue una experiencia agradable para los estudiantes, quienes ahora buscan comprender y participar de manera positiva en su grupo recordándose unos a otros lo importante que es el respeto, el diálogo, la escucha y el manejo de las emociones, entre otras habilidades estrechamente relacionadas con las competencias ciudadanas.

Palabras clave: competencias ciudadanas; enseñanza de competencias ciudadanas; convivencia escolar; ambientes lúdicos; talleres pedagógicos.

\title{
Strengthening citizenship competencies by means of ludic environments
}

\begin{abstract}
This work was born as an alternative to observing a weakened school coexistence among second-grade students of an educational institution. During the diagnostic stage, we determined that this situation was taking place due to the lack of use of the skills conducive to a peaceful coexistence, so it is necessary to strengthen the citizenship skills in students, understanding that these are a set of skills that, related to knowledge, allow training individuals that can not only recognize their environment but also, how to relate to it. To achieve this objective, we resorted to using ludic environments, defined as spaces that allow interaction among people and motivate their expression, fun, and reflection. We carried out the strategy by planning and implementing pedagogical workshops that are subsequently analyzed through field diaries, in order to recognize any progress made. Organizing ludic environments by the agency of different pedagogical workshops was enriching, because during the development of these activities, we observed situations of disagreement and discussions that were solved at the hand of dialogue, reflection, and teamwork, which are necessary skills in citizenship competencies. Finally, we found that this was a pleasant experience for the students, who now seek to understand and engage positively in their group, reminding each other of the importance of respect, dialogue, listening and managing emotions, among other skills closely related to citizenship competencies.
\end{abstract}

Keywords: citizenship competences; teaching of citizenship competences; school coexistence; ludic environments; pedagogical workshops. 


\section{Fortalecendo as habilidades cidadãs através de ambientes lúdicos}

\section{Resumo}

O presente trabalho nasceu como uma alternativa ao observar uma convivência escolar fragilizada entre alunos do segundo ano de uma instituição de ensino. Durante a fase de diagnóstico foi constatado que isso ocorria pela falta de utilização das suas próprias habilidades para a convivência pacífica, por isso se estabelece que é necessário fortalecer as habilidades cidadãs dos alunos, entendendo que se trata de um conjunto de habilidades que, relacionadas ao conhecimento, permitem a formação de indivíduos capazes de reconhecer o seu meio e a forma de se relacionar com ele. Para atingir este objetivo, buscou-se a utilização de ambientes lúdicos, definidos como espaços que permitem a interação entre as pessoas e motivam a expressão, a diversão e a reflexão. A estratégia foi implementada por meio do planejamento e aplicação de oficinas pedagógicas que são analisadas por meio de diários de campo, a fim de reconhecer os avanços alcançados neles. Organizar ambientes lúdicos por meio de diferentes oficinas pedagógicas foi enriquecedor, visto que durante o desenvolvimento dessas atividades foram observadas situações de desentendimento e discussões que foram resolvidas por meio do diálogo, da reflexão e do trabalho em equipe, competências necessárias nas habilidades cidadãs. Finalmente, verificou-se que esta foi uma experiência agradável para os alunos, que agora buscam compreender e participar de forma positiva em seu grupo, lembrando uns aos outros da importância do respeito, do diálogo, da escuta e da gestão das emoções, entre outras habilidades de aproximação relacionadas às habilidades cidadãs.

Palavras-chave: habilidades cidadãs; ensino de habilidades cidadãs; convivência escolar; ambientes lúdicos; oficinas pedagógicas.

\section{Renforcement des compétences civiques grâce à des environnements ludiques}

\section{Résumé}

Cette étude représente une alternative née de l'observation d'une coexistence détériorée entre élèves de secondaire d'un établissement scolaire. Une première phase de diagnostic a permis d'établir qu'il s'agissait d'une mauvaise utilisation des capacités de coexistence pacifique des élèves et qu'il convenait de renforcer leurs compétences civiques. Une fois reconnu et assimilé, cet ensemble de compétences permet de former des individus capables de reconnaître leur environnement et de s'y rapporter. Pour atteindre cet objectif, nous avons mis en place des environnements ludiques, définis comme des espaces permettant des interactions entre élèves, facilitant l'expression, le plaisir et la réflexion. La stratégie mise en place est réalisée au travers d'une planification et d'une réalisation d'ateliers pédagogiques analysés au travers de carnets de notes dans lesquels sont retranscrits les progrès réalisés. Cette organisation d'espaces ludiques et d'ateliers pédagogiques s'est révélée enrichissante car lors de ces activités, des situations de désaccords et de discussions ont été observées et se sont résolues par le dialogue, la réflexion et le travail en équipe - compétences nécessaires aux relations entre individus. Enfin, cette expérience agréable pour les étudiants, les pousse désormais à se comprendre et à participer de manière constructive dans leur groupe en se rappelant l'importance du respect, du dialogue, de l'écoute et de la gestion des émotions en fonction des compétences civiques.

Mots-clés: compétences civiques; enseignement des compétences civiques; vie scolaire; environnements ludiques; ateliers pédagogiques. 


\section{Introducción}

El proyecto «Fortaleciendo las competencias ciudadanas a través de ambientes lúdicos» se origina al evidenciar que los estudiantes de segundo grado del Instituto de Pedagogía Auto-activo de Grupos IPAG presentan actitudes irrespetuosas y poco tolerantes, escasa comunicación asertiva y un manejo de sus emociones que afecta su convivencia escolar. Al comprender que las competencias ciudadanas son un conjunto de habilidades cognitivas, emocionales y comunicativas (Chaux, 2012) necesarias para el desarrollo integral del ser humano y la convivencia pacífica, se propone fortalecer estas competencias a través de ambiente lúdicos que permitan la interacción, el disfrute y la reflexión de los individuos que participan en estos espacios.

Lo anterior cobra valor al reconocer que las competencias ciudadanas deben ser fortalecidas desde una temprana edad, pues a partir de estas los individuos construyen su identidad y se reconocen como parte de una sociedad; en palabras de Chaux, Lleras y Velásquez (2004), mediante la configuración de habilidades sociales las personas tienen una visión de sí mismos como individuos y miembros sociales. Es por esta razón que para el desarrollo de este proyecto se tiene en cuenta una serie de etapas, organizadas por capítulos, en los cuales se presenta la descripción del problema, los objetivos, el marco referencial, la metodología a seguir, los resultados obtenidos en relación con la aplicación de la estrategia - teniendo en cuenta cada una de las fases investigativas-y los instrumentos utilizados en estas, entre otros aspectos de la investigación.

En los últimos capítulos de este proyecto se establecen las conclusiones y las recomendaciones, apartados que permiten establecer una visión general de lo que fue el desarrollo del proyecto y lo que debería tenerse en cuenta para el futuro, reconociendo en ellos que las competencias ciudadanas son destrezas que permanecen a lo largo de la 
vida y se convierten en herramientas para la solución de problemas y la construcción de una convivencia pacífica.

\section{Fundamentación teórica}

En el desarrollo de este proyecto se procedió a evidenciar las categorías escogidas (Tabla 1), junto con un autor representativo de estas, con el fin de interpretar de forma adecuada autores que, con sus conocimientos y experiencias sobre estos temas, enriquecen el proyecto.

Tabla 1. Categorías de análisis de fundamentación teórica

\begin{tabular}{|l|l|}
\hline \multicolumn{1}{|c|}{\begin{tabular}{c}
\multicolumn{1}{|c|}{ Categoría 1 } \\
competencias ciudadanas
\end{tabular}} & \multicolumn{1}{|c|}{$\begin{array}{c}\text { Categoría 3 } \\
\text { ambientes lúdicos }\end{array}$} \\
\hline $\begin{array}{l}\text { Chaux, Lleras y Velásquez (2004) } \\
\text { Ruiz-Silva y Chaux-Torres (2005) } \\
\text { Chaux (2012) } \\
\text { Audigier (2000) }\end{array}$ & Bernard (1925) \\
\hline
\end{tabular}

Fuente. Elaboración propia.

Para la fundamentación teórica se tienen presentes los autores organizados en la tabla1; quienes nutren y fundamentan el proyecto. En este documento se realiza una síntesis de la fundamentación teórica.

\subsection{Competencias ciudadanas}

En Colombia se busca que los estudiantes se formen de manera integral para que sean ciudadanos que den cuenta de las habilidades que se obtienen gracias al desarrollo de las competencias ciudadanas; reconocidas como las destrezas orientadas a la construcción de personas autónomas que reconocen a los demás como seres de derechos y deberes que merecen ser respetados y protegidos (Audigier, 2000). En ese orden de ideas, Chaux 
(2012) sostiene que «las competencias ciudadanas son capacidades cognitivas, emocionales y comunicativas que integradas entre sí y relacionadas con conocimientos y actitudes, hacen posible que el ciudadano actué de manera productiva en la sociedad». En relación con lo anterior, desde el enfoque educativo, se hacen presentes con el fin de adquirir habilidades con las que se dé soluciones pacíficas a las problemáticas que puedan surgir dentro de la convivencia con los demás.

De acuerdo con Ruiz-Silva y Chaux-Torres (2005), las competencias ciudadanas permiten que se forme una sana convivencia y esto se logra cuando el individuo es capaz de alcanzar capacidades cognitivas, emocionales y comunicativas que le ayuden a comprender, respetar y comunicarse con los demás; por tanto, divide «las competencias ciudadanas en cuatro tipos, emocionales, cognitivas, comunicativas e integradoras» (p. 16), así mismo, cada una de estas tienen unas subcompetencias que deben ser abordadas para que cada individuo fortalezca suscompetencias.

\subsection{Convivencia escolar}

Antes de comprender de forma adecuada qué es la convivencia escolar, es necesario reconocer qué es la convivencia, entendiéndose como vivir juntos. Con respecto a este concepto Ortega (2007, p. 50) afirma lo siguiente:

El término convivencia encierra todo un campo de connotaciones y matices cuya suma revela la esencia que vincula a los individuos y que les hace vivir armónicamente en grupo. Se trata de ceñirse a unas pautas de conducta que permiten la libertad individual al tiempo que salvaguardan el respeto y la aceptación de los otros.

Básicamente, indica que la convivencia es el estado en el que se realiza una interacción con otras personas con las cuales sé es capaz de vivir armónicamente bajo reglas y principios básicos. En este sentido, la convivencia escolar se entiende como «el 
establecimiento de relaciones interpersonales y grupales satisfactorias que contribuyan a un clima de confianza, respeto y apoyo mutuo en la institución escolar» (Ortega, 2007). La convivencia escolar es, entonces, un factor importante en el desarrollo integral de las personas, pues a través de esta se alcanzan ciertos valores y habilidades necesarios para la vivencia en armonía entre los compañeros y que a futuro servirán, puesto que el ser humano es social por naturaleza y se enfrenta a convivir con otros a lo largo de su vida.

Ahora bien, es necesario tener en cuenta que la convivencia escolar positiva permite que los estudiantes se motiven a crecer con relaciones de convivencia pacífica, mejoren sus desempeños académicos y alcancen destrezas en la resolución de conflictos, entendiendo que son miembros activos de una comunidad (Ortega, 2007). Este pensamiento no se encuentra solo referenciado por Ortega, pues en términos simples la convivencia escolar hace referencia a «la calidad de relaciones en el que se promueve el reconocimiento mutuo, el respetoy el diálogo, promoviendo el desarrollo de la comunidad educativa y la construcción de la ciudadanía» (Secretaría de Educación del Distrito, 2019).

\subsection{Ambientes lúdicos}

De la misma forma que se realizó con la categoría de convivencia escolar, en esta categoría es necesario tener un conocimiento sobre qué es la lúdica. Este concepto está relacionado con la necesidad del ser humano de expresarse en formas diferentes en las que pueda involucrar emociones y vivencias, fomentando de ese modo la personalidad. En este orden de ideas se entiende como:

Una dimensión del desarrollo humano, siendo parte constitutiva del mismo, que se refiere a la necesidad del ser humano, de comunicarse, sentir, expresarse y producir sensaciones orientadas hacia el entretenimiento, la diversión, el esparcimiento, que pueden llevarnos a gozar, reír, gritar o inclusive llorar en una verdadera manifestación de emociones, que deben ser canalizadas adecuadamente por el facilitador del proceso. (Bernard, 1925) 
Lo anterior se toma como un bosquejo acerca de cómo la lúdica fomenta el desarrollo psicosocial, la adquisición de saberes y brinda herramientas para consolidar la personalidad, todo através de una amplia gama de posibilidades en las que interactúan el gozo, el placer, la creatividad y el conocimiento. Es por esto que, a través de «la generación de ambientes lúdicos, se estimula a los seres humanos creando espacios propicios para el aprendizaje» (Yturralde, 2001).

Los ambientes lúdicos son espacios que permiten la interacción entre las personas y abren la posibilidad de, a través de diversas actividades, promover el sentir de emociones y que estas puedan ser expresadas. Además, permiten la reflexión y el aprendizaje, ya que son «importante[s] para el aprendizaje experiencial y significativo puesto que dentro de estos espacios se producen emociones, gozo, placer y ambientes agradables de convivencia y participación» (Yturralde, 2001).

Con respecto a lo anterior, es valioso mencionar que los ambientes lúdicos son una estrategia significativa en relación con el objetivo planteado para el presente proyecto dado que llevan a interactuar naturalmente mediante una conciencia clara de colectividad y reflexión a través de las experiencias y vivencias que dentro de estos espacios se posibilitan.

\section{Metodología}

En la tabla 2 se presentan los elementos metodológicos aplicados en el proceso del proyecto investigativo, los cuales dan forma general y aportan al proceso del fortalecimiento. Con miras a la necesidad encontrada estos elementos metodológicos se dividen tal como se muestra. 
Tabla 2. Aspectos fundamentales de la metodología

\begin{tabular}{|l|l|l|}
\hline Aspecto & Descripción & Autores que fundamentan \\
\hline Enfoque de la investigación & $\begin{array}{l}\text { Estilos y las prácticas de la } \\
\text { investigación cualitativa. }\end{array}$ & $\begin{array}{l}\text { Hernández-Sampieri, Fernández- } \\
\text { Collado y Baptista-Lucio, 2003 }\end{array}$ \\
\hline Tipo de la investigación & $\begin{array}{l}\text { El tipo de investigación de este } \\
\text { proyecto es la investigación-acción. }\end{array}$ & $\begin{array}{l}\text { Restrepo, B, } 2004 \\
\text { Elliott, J. (2000 } \\
\text { Lewin, K. (1946 }\end{array}$ \\
\hline
\end{tabular}

Fuente. Elaboración propia.

El enfoque investigativo del presente proyecto es un componente de diseño cualitativo, debido a que cuenta con una flexibilidad e interactividad en el proceso de investigación. Esto incluye el análisis profundo y reflexivo de los datos recogidos a través de métodos específicos tales como la observación, la entrevista y los diarios de campo, entre otros, los cuales permiten conocer el significado de las acciones humanas y de la vida social de la población. Así mismo, va de la mano con el tipo de investigación acción que es concebida como una actividad en bien de todos, consistente en una práctica social en la que interactúa la teoría y la práctica con miras a establecer cambios apropiados a una realidad estudiada.

Por otra parte, teniendo en cuenta el tema de la investigación que corresponde a fortalecer las competencias ciudadanas para mejorar la convivencia escolar, permite que tenga relación con la línea de investigación de aula y escuela, cuyo objetivo principal es desarrollar el aprendizaje y que se amplíe el radio de acción pedagógica a partir de las problemáticas encontradas en contextos educativos. 
Tabla 3 . Población y muestra

\begin{tabular}{|l|l|}
\hline \multicolumn{1}{|c|}{ Aspecto } & \multicolumn{1}{|c|}{ Breve descripción } \\
\hline Población & En el 2019 el IPAG. \\
\hline Muestra & $\begin{array}{l}\text { Está constituida en el } 10,5 \% \text { con relación a la población } \\
\text { educativa perteneciente al grado segundo con una cantidad } \\
\text { de veinticuatro estudiantes. }\end{array}$ \\
\hline Contexto & $\begin{array}{l}\text { El IPAG tiene una población } 240 \text { estudiantes organizados de } \\
\text { grado jardín hasta grado once, ubicados en una sola sede; se } \\
\text { encuentran en un nivel socioeconómico de estrato } 2 \text { y 3; la } \\
\text { totalidad de los educandos viven en el mismo sector donde se } \\
\text { encuentra la institución. Esta institución es de origen privado } \\
\text { desde } 1966 \text { hasta la fecha. }\end{array}$ \\
\hline
\end{tabular}

Fuente. Elaboración propia.

\subsection{Fases de investigación}

En la tabla 4 se encuentran los verbos que clasifican los objetivos de la investigación planteada, junto con su debido instrumento, que globalmente conforman la fase de la investigación.

Tabla 4. Fases de la investigación

\begin{tabular}{|l|l|l|}
\hline Fase de investigación & $\begin{array}{l}\text { Objetivo } \\
\text { específico }\end{array}$ & Instrumento \\
\hline Diagnóstico & Identificar & Entrevista \\
\hline Trabajo de campo & Organizar & Talleres pedagógicos \\
\hline Reflexión & Sintetizar & Diarios de campo \\
\hline
\end{tabular}

Fuente. Elaboración propia.

Los instrumentos empleados en este proyecto generan un aporte apropiado para dar un avance positivo a la necesidad encontrada, sin dejar de lado la secuencia de la investigación. Las entrevistas, junto con la observación, ayudarán a ver la problemática abordada que, con la aplicación de la estrategia implementada en los talleres 
pedagógicos, se genera en un ambiente significativo que permite captar y mejorar la disposición de la población tomada como muestra.

Así, el escrito debe presentar una estructura coherente y ordenada, de manera que permita unir las ideas a través de un hilo conductor. También debe tener una redacción clara que permita comprender lo que se quieren trasmitir.

\section{Análisis de resultados}

\subsection{Fase diagnostica. Entrevista a docentes}

Teniendo en cuenta la información recolectada en la tabla 3 se hace necesario realizar un análisis de las respuestas dadas por los entrevistados. Cabe destacar que esta entrevista ha sido realizada específicamente a los docentes que prestan sus servicios laborales en el grado segundo, teniendo en cuenta que, a través de la observación, las investigadoras han encontrado una problemática en el uso de sus competencias ciudadanas, estableciendo la entrevista como método de diagnóstico y confirmación de la problemática que hallada.

Para el desarrollo de la entrevista se realizaron ocho preguntas en las que se buscó reconocer la perspectiva que tienen frente a la convivencia y el actuar de los estudiantes de segundo grado. En ese orden de ideas se establece que, de acuerdo con el punto de vista, los docentes consideran que los estudiantes durante las clases realizan las actividades y tratan de mantener una participación en estas. Sin embargo, esto no sucede siempre, pues existen estudiantes que tienden a burlarse de las respuestas equivocadas que los demás pueden dar.

Por otra parte, entre sus respuestas mencionan que las discusiones en el grupo se presentan por diferencias de pensamientos, lo que les dificulta las actividades en grupo, pues inevitablemente terminarán en discusiones y comentarios ofensivos. Si bien estas 
acciones han sucedido desde el año anterior, no era marcado su comportamiento en relación con este año, cuando incluso toman posturas agresivas e intolerantes.

La información recolectada también permite comprender que los docentes y la institución, en general, tienen claros parámetros o conductos a seguir cuando se presentan situaciones problema en los estudiantes, de acuerdo con su levedad o gravedad. Por medio de estos conductos se tiene en cuenta involucrar entes tales como la psicoorientadora, la coordinación y los padres de familia; de estos últimos no siempre se obtieneel apoyo adecuado, por lo que el estudiante terminará por aumentar su agresividad y su comportamiento negativo.

Teniendo en cuenta lo anterior, se puede concluir que los comportamientos que se presentan entre los estudiantes se deben a la falta de fortalecer habilidades como, por ejemplo, la escucha activa, el asertividad y la perspectiva, entre otras destrezas que se encuentran directamente relacionadas con las competencias ciudadanas. Por tanto, se hace necesario fortalecer estas competencias, lo que permitirá que su clima escolar mejore; además de convertirlas en componentes de su formación integral, lo que a futuro le hará posible que los estudiantes se proyecten como personas capaces de establecer relaciones sanas y convivir adecuadamente en sociedad.

\subsection{Fase de trabajo de campo y reflexión. Diario de campo}

Si se tienen en cuenta cada uno de los diarios de campo realizados por cada uno de los talleres, es necesario realizar un análisis en relación con lo que en ellos puede constatarse. En este sentido, es necesario mencionar que cada uno de los talleres refleja en su aplicación las distintas habilidades, con miras a que en la vida real es imposible separar los distintos tipos de competencias, pues los seres humanos son un compendio de emociones, pensamientos, gestos y palabras. 
El desarrollo de los talleres permitió concientizar a los estudiantes sobre la forma en la que actúan entre ellos y con los demás. De esta manera, es necesario mencionar que estos procesos sedieron gracias a que se propiciaron ambientes en los que los estudiantes, en realidad, se verían motivados a participar, convivir, comunicar y colaborar entre ellos para obtener buenos resultados en las diferentes actividades que se les propuso desarrollar.

Por otra parte, es cierto que no todo es perfecto, pues de estas interacciones, por supuesto, surgieron indiferencias, discusiones e, incluso, ofensas. Sin embargo, fue fundamental que las investigadoras estuvieran pendientes de estos procesos y fomentaran el análisis y la reflexión de las actitudes, no solo propias, sino de los demás, cuando sucedían estas situaciones, de manera que los mismos miembros de este curso participaran y promovieran acciones más respetuosas o aconsejaran técnicas para el manejo de emociones como la ira.

En ese orden de ideas es necesario mencionar que en la escolaridad es realmente fundamental realizar este tipo de espacios en los que la convivencia, el análisis y la reflexión vayan de la mano. Pues, permiten que los estudiantes sean conscientes de su clima escolar, las situaciones que lo nublan y las formas de mejorar no de manera individual, sino conjunta. El desarrollo de estos talleres permitió visualizar que las competencias ciudadanas son un componente necesario en la convivencia de cualquier tipo y, en el caso del grado segundo, establecieron pautas que deben continuar siendo fortalecidas para que alcancen la formación integral que los pilares de la educación se proponen. 


\section{Conclusiones}

En relación con los ambientes lúdicos, estos fueron una estrategia acertada, puesto que, como se menciona en el marco teórico, son espacios que promueven ambientes agradables de convivencia basados en la participación activa, los valores éticos y la reflexión que, relacionados con fortalecer las competencias ciudadanas, permitieron que los estudiantes tomaran conciencia como parte de una comunidad en la que se debe ser un miembro que aporte a la armonía, al buen trato y al respeto.

En la fase diagnostica se utilizó un instrumento que permitió establecer y dar soporte a la investigación, pues observar en los estudiantes las competencias ciudadanas en relación con su convivencia escolar y encontrar que faltaba hacer uso de estas habilidades fue la causa por la que se buscó obtener las perspectivas desde el punto de vista de los docentes que día a día conviven con los estudiantes. La información obtenida fue fundamental para determinar la meta establecida a través de cada uno de los objetivos, entendiendo este tipo de competencias son esenciales en la vida del ser humano como parte del saber ser y convivir sanamente.

En este sentido, organizar ambientes lúdicos mediante distintos talleres pedagógicos, en los que el objetivo fue siempre motivar al cambio y la reflexión para fortalecer las habilidades de los estudiantes, resultó enriquecedor. Durante el desarrollo de estas actividades se observaron situaciones de desacuerdo y discusiones, pero fue posible solucionarlas a través del diálogo, la reflexión y el trabajo en equipo, destrezas necesarias en las competencias ciudadanas que, como se ha mencionado a lo largo de la investigación, están relacionadas con lo cognitivo, lo emocional y lo comunicativo. 
Por lo anterior, fue necesario sintetizar lo que se realizó en cada taller y el resultado que se obtuvo mediante los diarios de campo, un instrumento adecuado que permitió la reflexión. Además,se constató que esta fue una experiencia agradable para los estudiantes, quienes ahora buscan comprender y participar de manera positiva en su grupo recordándose unos a otros lo importante que es el respeto, el diálogo, la escucha y el manejo de las emociones, entre otras habilidades que se encuentran estrechamente relacionadas con las competencias ciudadanas.

Esta investigación fue un proyecto enriquecedor que le permitió a las investigadoras acercarsea un tema que es fundamental en el desarrollo integral de todos los seres humanos, pues la educación no se basa solo en el aprendizaje cognitivo y procedimental, sino que también debe tener en cuenta el lado humano y social basado en la formación de personas que reconozcan cómo actuar de manera justa y responsable, solucionen sus conflictos, se relacionen pacíficamente y aporten de forma positiva a la grupos sociales a los que pertenece, porque, como menciona Chaux, Lleras y Velásquez (2004), los ciudadanos competentes son capaces no solo de reconocerse y valorarse, sino también a los demás para vivir en sociedad.

\section{Referencias}

Audigier, F. (26 de junio del 2000). Basic concepts and core competencies for education for democratic citizenship. Estrasburgo, Francia. Recuperado de https://bit.ly/3bADzwN

Bernard, G. (1925). Dimensión del desarrollo humano. Madrid: Ediciones Cátedra.

Chaux, E. (2012). Educación, convivencia y agresión escolar. Bogotá: Aguilar; Universidad de los Andes. DOI: https://doi.org/10.7440/2012.62

Chaux, E.; Lleras, J.; Velásquez, A. (2004). Competencias ciudadanas: de los estándares al aula. Bogotá: Universidad de los Andes. DOI: https://doi.org/10.7440/2004.01 
Elliott, J. (2000). La investigación-acción en educación. Madrid: Morata.

Hernández-Sampieri, R.; Fernández-Collado, C.; Baptista-Lucio, M. (2003). Metodología de la investigación. México: Mc Graw-Hill.

Lewin, K. (1946). La investigación-acción y los problemas de las minorías. En M.C. Salazar, (Comp) (1992). La Investigación acción participativa. Inicios y Desarrollos. (13 -25). Madrid: Editorial Popular OEI.

Ortega, R. (2007). La convivencia: un regalo de la cultura a la escuela. Idea La Mancha, (4) 50-54.

Ruiz-Silva, A.; Chaux-Torres, E. (2005). Las competencias ciudadanas. En La formación de competencias ciudadanas. (28-67). Bogotá: Ascofade.

Secretaría de Educación del Distrito. (8 de febrero de 2019). Convivencia escolar. Recuperado de https://bit.ly/3jMl4tu

Yturralde, E. (2001). Metodologías participativas. Recuperado de https://bit.ly/3nB3SZ3 